Revue d'études américaines. American Studies Journal

\title{
Réception du 11 septembre 2001 au Québec. Persistance des mémoires nationales et émergence de mémoires globalisées
}

\section{Christian Bergeron}

\section{CpenEdition}

Electronic version

URL: https://journals.openedition.org/transatlantica/7448

DOI: $10.4000 /$ transatlantica.7448

ISSN: $1765-2766$

Publisher

Association française d'Etudes Américaines (AFEA)

Electronic reference

Christian Bergeron, "Réception du 11 septembre 2001 au Québec. Persistance des mémoires nationales et émergence de mémoires globalisées", Transatlantica [Online], 1 | 2015, Online since 12 January 2016, connection on 03 February 2023. URL: http://journals.openedition.org/transatlantica/ 7448 ; DOI: https://doi.org/10.4000/transatlantica.7448

This text was automatically generated on 3 February 2023

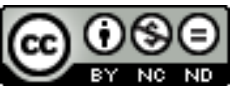

Creative Commons - Attribution-NonCommercial-NoDerivatives 4.0 International - CC BY-NC-ND 4.0 https://creativecommons.org/licenses/by-nc-nd/4.0/ 


\title{
Réception du 11 septembre 2001 au Québec. Persistance des mémoires nationales et émergence de mémoires globalisées
}

\author{
Christian Bergeron
}

J'ai obtenu une bourse d'excellence du Conseil de recherches en sciences humaines du Canada (CRSH) pour la réalisation de ma thèse doctorale en sociologie (2013). Je tiens à remercier mon directeur de thèse, monsieur Daniel Mercure de l'Université Laval, ainsi que messieurs Christian Lalive d'Epinay et Stefano Cavalli (responsables de l'étude internationale CEVI).

\section{Introduction}

1 Tout le monde (ou presque) se souvient de cette date fatidique - de ces images troublantes diffusées en boucle par les médias ${ }^{1}$ - associée à la chute des deux tours du World Trade Center de New York, des attaques terroristes à Washington et en Pennsylvanie. Nous nous remémorons tous (ou presque) de nos activités menées au cours de cette journée et de la manière dont nous avons pris connaissance de cette nouvelle. Comparativement à d'autres événements sociohistoriques, le 11 septembre 2001 exprime en images télévisuelles des scènes brutales et inattendues par rapport à l'expérience de la vie quotidienne de millions d'individus. Ces attaques terroristes montrent "à la fois l'évidence d'une rupture et l'incertitude quant à sa signification " (Bensa et Fassin, 2002, 14). Ainsi, il devient pertinent, dans ce contexte, de comprendre la représentation de cet événement au Québec, dans un contexte de globalisation. D'une manière générale, la globalisation se caractérise par des États-nations, des territoires et des cultures locales en interdépendance et parfois en lutte, à l'échelle mondiale, dans des échanges d'activités économiques, technologiques, médiatiques, sociales, culturelles, politiques et humaines (Appadurai, 2001; Dubet, 2005 ; Eisenstadt, 2000 ; 
Sorensen et Christiansen, 2013). D'une manière plus précise, la globalisation peut se définir comme :

l'extension continue du capitalisme et du marché sans que celui-ci semble être aux mains d'un État national et d'une bourgeoisie nationale, [le] développement conjoint d'une culture de masse cosmopolite et des affirmations identitaires, [le] développement du principe de l'égalité démocratique, [1']affaiblissement des souverainetés politiques, [et le] déclin des formes institutionnelles de la socialisation... (Dubet, 2005, 6).

2 En d'autres mots, la globalisation peut être comprise comme un processus multidimensionnel qui influence les aspects culturels et politiques des pays à l'échelle mondiale (Sorensen et Christiansen, 2013). La dimension culturelle est influencée par les avancées technologiques, les multiples moyens médiatiques et visuels pour atteindre "le plus de monde possible ", au même moment, mais dans différents lieux géographiques et culturels. Appadurai $(2001,30)$ a démontré dans son ouvrage les liens «entre les événements transmis par les médias et les publics en déplacement ». Cet auteur s'est efforcé d'expliquer les liens entre la globalisation et la modernité avancée : «les moyens de communication électroniques et les migrations de masse s'imposent ainsi aujourd'hui comme des forces nouvelles [...]. Ensemble, ils créent des décalages spécifiques dans la mesure où les téléspectateurs circulent en même temps que les images » (Appadurai, 2001, 29). Cette circulation des individus et des images est rendue possible par le travail d'imagination des sociétés déterritorialisées (cf. Deleuze et Guattari, 1980). De fait, «la planète est un territoire doté d'une texture de communications (avions, phone, fax, Internet) comme jamais aucune société n'a pu en posséder dans le passé » (Morin, 2009, 19). Ce qui a pour effet que la planète «tend à s'unifier, à se "mondialiser", à susciter des formes de représentations collectives et d'actions publiques qui, au moins en apparence, se ressemblent de plus en plus» (Rousso, 2007, 3). Toutefois, il faut noter que la globalisation ne détruit pas totalement les frontières et les identités nationales, car «la globalisation constitue en elle-même un processus profondément historique, irrégulier, et qui renforce même l'ancrage dans le local » (Appadurai, 2001, 49). Bref, la globalisation s'enchevêtre, à différents niveaux, avec les sociétés locales :

Il y a, d'un côté, ce que nous sommes dans la réalité, et ce que nous devenons sous l'effet de la mondialisation culturelle, à savoir des êtres tissés de fils de toutes les couleurs, qui partagent avec la vaste communauté de leurs contemporains, l'essentiel de leurs croyances. Et puis il y a, d'autre part, ce que nous pensons être, ce que nous prétendons être, c'est-à-dire des membres de telle communauté et pas de telle autre (Maalouf, 1998, 137).

3 Paradoxalement, la globalisation amène les sociétés à se singulariser, " parce qu'elles ne sont plus perçues comme les déclinaisons locales d'un même modèle et comme les étapes successives d'une évolution toute tracée » (Dubet, 2009, 325).

Les événements du 11 septembre 2001, aux États-Unis, expriment clairement cette réflexion distancée, ce travail réflexif de l'individu. Ces événements s'enracinent dans un contexte de déterritorialisation et de globalisation. Les effets de la globalisation, amplifiés par l'essor des nouvelles technologies d'information et de communication, sont des éléments essentiels pour la conduite d'une réflexion distancée de cet événement diffusé en direct dans plusieurs pays. Par exemple, les mêmes images des attaques terroristes ont envahi nos écrans, comme jamais un événement historique ne l'a fait auparavant (Niemeyer, 2010). La globalisation permet l'émergence « d'un nouvel espace public mondial» (Rousso, 2007, 5) vécu à l'intérieur de temporalités sociales 
comparables. Dans ce sens, le 11 septembre 2001 « symbolise à l'excès certains traits de la globalisation actuelle " (Lalive d'Epinay et coll., 2012, 7) tout en stimulant la construction d'une histoire mondiale partagée (Rousso, 2007). Dans cet article, nous étudions au Québec l'impact et la signification du 11 septembre 2001 chez différentes cohortes d'âge. De cette manière, nous observerons l'importance de la globalisation dans leur soi historique.

Le soi historique ou la conscience générationnelle (Mannheim, 1990 [1928]) se construit individuellement en prenant conscience des événements sociohistoriques. Nous pouvons parler, à juste titre, d'une individualisation du soi historique. Mannheim (1990 [1928] avance que le processus de la conscience générationnelle chez les individus se fixe pendant l'adolescence. Autrement dit, les premières impressions du monde constituent le noyau autour duquel s'articuleront les impressions ultérieures. Quant à Martuccelli $(2006,236)$, le soi historique "est la façon dont certains individus se comprennent eux-mêmes comme un produit de l'histoire et en même temps comprennent le sens liminaire de l'histoire à l'échelle de leurs vies. »

\section{Questions de recherche}

6 La déterritorialisation s'observe dans le domaine social, économique et politique des sociétés contemporaines. Par exemple, la séparation spatio-temporelle de nos relations collectives amène une nouvelle dynamique où les mécanismes de délocalisation fournissent les moyens de dissocier «les relations sociales de leur localisation en des lieux spécifiques» (Giddens, 1994, 59) et de permettre ainsi aux individus de s'approprier la connaissance par la réflexion distancée. Premièrement, «dans quelle mesure [constatons-nous] la persistance de mémoires collectives avant tout composées d'épisodes situés dans le cadre de l'État-nation?» (Lalive d'Epinay et coll., 2008, 3). Deuxièmement, dans quelle mesure constatons-nous l'émergence d'éléments de la globalisation et de la déterritorialisation (cf. Appadurai, 2001; Beck, 2000 ; Giddens, 1994) dans le soi historique des individus?

\section{Matériel Empirique}

7 L'étude internationale CEVI (Changements et événements au cours de la vie), dirigée par Lalive d'Epinay et Cavalli (en cours²), analyse le déroulement des vies individuelles dans leur relation avec le contexte sociétal et historique, en prenant pour révélateur la notion d' "événement marquant de la vie » ainsi que la perception qu'en a l'individu. L'étude, reprise dans plusieurs pays, comprend trois volets : 1) les changements récents dans la vie individuelle (dans l'année écoulée), 2) les principaux tournants survenus dans la vie individuelle et 3) les principaux changements et événements sociohistoriques survenus à l'échelle nationale ou locale, d'une part, et à l'échelle globale, d'autre part, depuis la naissance de l'individu. Dans le cadre de cet article, nous présenterons uniquement les résultats quantitatifs et qualitatifs du volet III pour le Québec. Le questionnaire standardisé, comportant des questions ouvertes et fermées, s'intitule Changements et événements au cours de la vie ${ }^{3}$. Le troisième volet à l'étude, intitulé Les événements et changements sociohistoriques, cherche à mieux comprendre "les contours de la dimension historique au sein de la mémoire collective propre à chaque pays ainsi que ses différentiations générationnelles ». La première question posée est 
celle-ci : «Considérons maintenant les grands événements et changements qui se sont produits dans votre pays (ou votre province) et dans le monde au cours de votre vie. Quels sont ceux qui vous ont le plus frappé ?» La personne interrogée doit faire une description de ces événements et ensuite répondre à la seconde question : «Pourquoi vous [ont]-t-il[s] marqué particulièrement?» et indiquer l'année, l'âge et le lieu au moment précis du changement. La personne interrogée peut mentionner jusqu’à quatre événements et changements sociohistoriques.

\section{La population à l'étude}

Afin de pouvoir effectuer ultérieurement des études comparatives, nous avons utilisé une méthodologie de recherche similaire à celle des autres équipes CEVI. L'objectif est d'atteindre de 500 à 600 participants comme dans les autres études internationales. Dans notre recherche canadienne, nous avons obtenu la participation de 504 personnes de l'agglomération urbaine de la Ville de Québec. L'échantillon de l'étude, nonaléatoire, est stratifié selon le sexe et selon cinq classes d'âges quinquennales séparées, chaque fois, par un intervalle de 10 ans (20-24, 35-39, 50-54, 65-69 et 80-84 ans), représentant des cohortes d'individus nés entre 1926 et 1990 . Le recrutement a été réalisé par 20 étudiants de premier cycle universitaire et nous-mêmes.

\section{Résultats}

En 2010, 504 participants de la Ville de Québec ont rempli le questionnaire CEVI. Les principales caractéristiques sociodémographiques sont présentées au tableau 1.

Tableau 1 : Caractéristiques de l'échantillon

\begin{tabular}{|l|l|l|l|l|l|}
\hline $\begin{array}{l}\text { Caractéristique } \\
\text { (Nombre de participants) }\end{array}$ & $\begin{array}{l}20-24 \\
(104)\end{array}$ & $\begin{array}{l}35-39 \\
(100)\end{array}$ & $\begin{array}{l}50-54 \\
(100)\end{array}$ & $\begin{array}{l}65-69 \\
(100)\end{array}$ & $\begin{array}{l}80-84 \\
(100)\end{array}$ \\
\hline $\begin{array}{l}\text { Âge moyen } \\
\text { Écart-type }\end{array}$ & 21,50 & 36,69 & 52,19 & 66,16 & 80,89 \\
2,91 & 2,63 & 2,99 & 3,33 \\
\hline Sexe féminin (\% col.) & 51 & 50 & 50 & 50 & 50 \\
\hline Lieu de naissance à Québec (\% col.) & 43 & 52 & 48 & 35 & 40 \\
\hline $\begin{array}{l}\text { État civil (\% col.) } \\
\text { Célibataires } \\
\text { Conjoint(e) de fait } \\
\text { Marié(e)s }\end{array}$ & 74 & 19 & 16 & 13 & 19 \\
Divorcé(e)s/séparé(e)s & 21 & 46 & 23 & 7 & 2 \\
Veufs/veuves & 4 & 28 & 44 & 60 & 32 \\
\hline A au moins un enfant (\% col.) & 2 & 6 & 17 & 14 & 4 \\
\hline
\end{tabular}




\begin{tabular}{|l|l|l|l|l|l|}
\hline Cohabitation (\% col.) & 12 & 14 & 21 & 30 & 50 \\
Seul & 21 & 73 & 66 & 66 & 33 \\
Partenaire & 40 & 3 & 4 & 0 & 0 \\
Parents & 27 & 10 & 9 & 4 & 17 \\
Membres de la famille/colocataires & & & & & \\
\hline Niveau d'éducation (\% col.) & 1 & 0 & 5 & 10 & 33 \\
École primaire & 6 & 20 & 33 & 26 & 28 \\
École secondaire/métier prof. & 43 & 27 & 19 & 22 & 11 \\
Cégep/collège classique & 50 & 53 & 43 & 42 & 28 \\
Universitaire & 86 & 9 & 2 & 0 & 0 \\
\hline Situation professionnelle (\% col.) \\
Étudiant \\
Marché du travail/chômeur \\
Foyer/assisté social \\
Retraité
\end{tabular}

10 Excepté les octogénaires ( $50 \%$ d'entre eux vivent seuls), les participants cohabitent avec un partenaire ou un colocataire (de $48 \%$ à $73 \%$ ). Aussi, ils sont majoritairement parents d'au moins un enfant (de $59 \%$ à $78 \%$ ), à l'exception des personnes de 20 à 24 ans $(2 \%)$. De plus, ils sont davantage scolarisés que la population québécoise. Chez les 20-24 ans et les 35-39 ans, 9 participants sur 10 ont une scolarité supérieure ou égale au niveau collégial ou universitaire, alors qu'au Québec c'est le cas de seulement 5 individus sur 10 (45\%) (Statistique Canada, 2006). En outre, chez les 80-84 ans, 4 participants sur 10 ont un niveau d'études supérieur ou égal au niveau collégial ou universitaire, alors que $27 \%$ des 65 ans et plus ont la même scolarité au Québec (Statistique Canada, 2006) .

\section{Analyses quantitatives}

11 Le tableau 2 présente les événements et changements sociohistoriques les plus mentionnés pour les cinq classes d'âge. L'événement le plus cité est celui du 11 septembre 2001 : il arrive en premier chez les trois premières classes d'âge $(29 \%, 22 \%$, $15 \%$ respectivement) et en deuxième chez les 65-69 ans (7\%). Toutefois, cet événement est presque absent des réponses des 80-84 ans (3\%). Attirons l'attention sur les deux référendums sur la souveraineté du Québec. En effet, nous remarquons que le dernier référendum de 1995 est quasiment inexistant chez les cohortes âgées de plus de 50 ans (8 mentions pour les 50-54 ans; 5 pour les 65-69 ans; et une pour les 80-84 ans). Également, la mention du premier référendum de 1980 est absente de chez les 65 ans et plus. C'est uniquement l'élection de René Lévesque, en $1976^{5}$, qui est mentionnée comme événement frappant pour les 50 ans et plus. Finalement, une grande diversité des changements sociohistoriques est mentionnée chez les participants comme en témoigne la catégorie « Autres » (de $51 \%$ à 67 \% des événements cités). 
Tableau 2 : Les événements et changements sociohistoriques les plus fréquents par classes d'âges

\begin{tabular}{|c|c|c|c|c|c|c|c|c|c|}
\hline \multicolumn{2}{|l|}{$20-24$ ans } & \multicolumn{2}{|l|}{ 35-39 ans } & \multicolumn{2}{|l|}{$50-54$ ans } & \multicolumn{2}{|l|}{$65-69$ ans } & \multicolumn{2}{|l|}{$80-84$ ans } \\
\hline Type & $\%$ & Type & $\%$ & Type & $\%$ & Type & $\%$ & Type & $\%$ \\
\hline 11 sept 01 & 29 & 11 sept 01 & 22 & 11 sept 01 & 15 & $\begin{array}{l}\text { Élection } \\
\text { du PQ } 76\end{array}$ & 8 & Seconde GM & 24 \\
\hline $\begin{array}{l}\text { Référendum } \\
95\end{array}$ & 8 & $\begin{array}{l}\text { Référendum } \\
95\end{array}$ & 11 & $\begin{array}{l}\text { Élection du } \\
\text { PQ } 76\end{array}$ & 8 & 11 sept 01 & 7 & $\begin{array}{l}\text { Révolution } \\
\text { tranquille }\end{array}$ & 7 \\
\hline $\begin{array}{l}\text { Élection } \\
\text { Obama } 08\end{array}$ & 4 & $\begin{array}{l}\text { Progrès* } \\
\text { comm. }\end{array}$ & 5 & $\begin{array}{l}\text { Référendum } \\
80\end{array}$ & 6 & $\begin{array}{l}\text { Progrès* } \\
\text { comm. }\end{array}$ & 6 & $\begin{array}{l}\text { Progrès* } \\
\text { comm. }\end{array}$ & 5 \\
\hline $\begin{array}{l}\text { Tempête de } \\
\text { verglas }\end{array}$ & 4 & $\begin{array}{l}\text { Élection } \\
\text { Obama } 08\end{array}$ & 4 & $\begin{array}{l}\text { Crise octobre } \\
70\end{array}$ & 6 & Mort JFK & 6 & $\begin{array}{ll}\text { Élection } & P Q \\
76 & \end{array}$ & 4 \\
\hline Haïti 10 & 4 & Haïti 10 & 4 & $\begin{array}{l}\text { Homme sur la } \\
\text { lune }\end{array}$ & 4 & $\begin{array}{l}\text { Crise } \\
\text { octobre } 70\end{array}$ & 6 & 11 sept 01 & 3 \\
\hline Autres & 51 & Autres & 54 & Autres & 61 & Autres & 67 & Autres & 57 \\
\hline Total & 100 & Total & 100 & Total & 100 & Total & 100 & Total & 100 \\
\hline
\end{tabular}

$(x 2=1058,94, p \leq 0,001)$

* Progrès dans les technologies de l'informatique et de communication.

12 Le tableau 3 présente les différents pays les plus cités pour les événements sociohistoriques les plus frappants par classe d'âge. Quant aux événements qui ont frappé le monde (plusieurs pays impliqués), ils se trouvent en troisième position chez les différentes cohortes et arrivent même en deuxième position chez les 80-84 ans. D'ailleurs, c'est la Seconde Guerre mondiale qui explique ce pourcentage élevé chez les octogénaires. Nous reviendrons plus loin sur l'importance des pays cités lors de la discussion des résultats.

Tableau 3 : Les lieux des changements sociohistoriques les plus fréquents par classes d'âges

\begin{tabular}{|l|l|l|l|l|l|l|l|l|l|}
\hline \multicolumn{2}{|l|}{$20-24$ ans } & \multicolumn{2}{l}{$35-39$ ans } & \multicolumn{2}{l}{$50-54$ ans } & \multicolumn{2}{l|}{$65-69$ ans } & \multicolumn{2}{l|}{$80-84$ ans } \\
\hline Lieu & $\%$ & Lieu & $\%$ & Lieu & $\%$ & Lieu & $\%$ & Lieu & $\%$ \\
\hline É.-U. & 38 & Québec & 34 & Québec & 43 & Québec & 48 & Québec & 44 \\
\hline Québec & 29 & É.-U. & 27 & É.-U. & 19 & É.-U. & 18 & Monde & 36 \\
\hline Monde & 9 & Monde & 13 & Monde & 14 & Monde & 17 & É.-U. & 5 \\
\hline Europe & 9 & Europe & 11 & Europe & 12 & Europe & 9 & Canada & 4 \\
\hline Canada & 6 & Afrique & 5 & Lune/espace & 5 & Afrique & 3 & Europe & 3 \\
\hline
\end{tabular}




\begin{tabular}{|l|l|l|l|l|l|l|l|l|l|}
\hline Autres & 8 & Autres & 9 & Autres & 7 & Autres & 5 & Autres & 8 \\
\hline Total & 100 & Total & 100 & Total & 100 & Total & 100 & Total & 100 \\
\hline
\end{tabular}

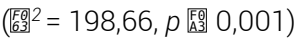

\section{Analyses qualitatives}

13 Le 11 septembre 2001 a été ressenti comme un basculement de mentalité : «nous avons changé d'époque, nous vivons désormais dans un monde différent » (Schmoll, 2002, 55). Le monde semble devoir s'ordonner autrement avec cet événement et il vient ainsi marquer « symboliquement la fin de l'époque moderne » (Schmoll, 2002, 55). Dans quel sens faut-il analyser cette rupture avec le monde moderne? Qu'est-ce qui se termine et qu'est-ce qui se commence avec le 11 septembre 2001 ? Premièrement, le fait que les États-Unis soient attaqués sur leur territoire marque l'imaginaire des populations occidentales. La superpuissance américaine n'est pas invincible et l'hégémonie idéologique américaine est lourdement frappée : «les tours du World Trade Center, le Pentagone et la Maison-Blanche, signent peut-être pour l'avenir la fin d'un ordre mondial que les Américains avaient pu croire consacrer par la chute du Mur de Berlin » (Schmoll, 2002, 55). Au lendemain du 11 septembre 2001, l'Amérique a perdu « l'illusion naïve de son splendide isolement, qu'elle croyait protégé par deux océans. Elle a découvert qu'elle n'avait plus de frontières protectrices " (Schmoll, 2002, 55). Certes, l'Amérique a perdu son sentiment de protection civile contre les conflits armés sur son territoire, mais c'est l'ensemble des civilisations occidentales qui ont réalisé leur vulnérabilité : « cela veut dire que si nous sommes certes assurés plus ou moins contre des accidents fortuits, nous sommes par contre exposés sans protection aucune aux attentats terroristes intentionnels " (Beck, 2003, 33). Pour ce qui est des participants de notre étude, ils ont aussi ressenti que le 11 septembre 2001 a marqué un basculement historique important. Les «intouchables " ou les «invincibles », comme le formulent deux participants, ont été attaqués au cœur de leurs symboles, ce qui signifie une certaine rupture sociohistorique au sein de la modernité avancée : "Je croyais que les Américains étaient intouchables. Donc de voir les avions frapper les tours et de voir l'impuissance des Américains à ce moment m'a rendu extrêmement triste " (homme de 66 ans, âgé de 57 ans en 2001) ; « Je suis américaine de cœur, j'ai réalisé qu'ils n'étaient pas invincibles... » (femme de 56 ans, âgée de 47 ans en 2001) ; "Acte terroriste nous rappelant que l'Amérique, jusque là épargnée, n'était plus à l'abri » (homme de 77 ans, âgé de 68 ans en 2001).

Les attaques terroristes provoquent la fin "de l'hégémonie et de l'invulnérabilité américaines et la sensation que les États-Unis incarnaient la dernière superpuissance au monde. Mais plus profondément encore, ces attentats ont mis fin au sentiment de sécurité et de confort des Américains" (Carr, 2010, 91). Ajoutons que ce sentiment d'insécurité n'affecte pas seulement les Américains, comme en témoignent ces extraits : " J'ai compris que personne n'est à l'abri du mal » (homme de 22 ans, âgé de 13 ans en 2001); "Ce qui se passe à l'étranger devient soudainement aussi notre affaire " (homme de 33 ans, âgé de 24 ans en 2001) ; "Nous sommes maintenant vulnérables » (homme de 50 ans, âgé de 41 ans en 2001); «Prendre conscience qu'on n'est pas en parfaite sécurité à aucun endroit » (homme de 33 ans, âgé de 24 ans en 2001) ; «Tout 
peut arriver à n'importe quel moment et nous pouvons mourir à tout moment " (femme de 19 ans, âgée de 10 ans en 2001) ; "Cette fois, la guerre était à notre porte, aux mains de fanatiques armés... aucune défense possible: toute la planète est maintenant en danger » (homme de 63 ans, âgé de 54 ans en 2001).

Le 11 septembre 2001 révèle le début d'un nouvel épisode de la modernité avancée avec une mondialisation du risque déterritorialisée (Sorensen et Christiansen, 2013, paraphrasant Beck). Cet événement amène "une nouvelle période historique plus dangereuse " (Fragnon, 2005, 4) et les participants prennent conscience de ce changement sociohistorique : «Les images étaient tellement fortes. Le monde a changé cette journée-là. J'ai senti l'urgence de vivre le moment présent " (femme de 34 ans, âgée de 25 ans en 2001); "Cet événement symbolise une rupture entre le monde idéalisé, sécuritaire et douillet de l'Amérique du Nord et la réalité » (homme de 26 ans, âgé de 17 ans en 2001) ; « Le début d'une nouvelle ère. L'ère de la peur, de la querelle, de la vengeance. La crainte qui va s'installer un peu tout partout. Plus rien n'est sûr " (femme de 41 ans, âgée de 32 ans en 2001) ; «J'ai eu l'impression que le monde allait radicalement changer » (homme de 33 ans, âgé de 24 ans en 2001).

\section{Discussion}

16 Les événements et changements sociohistoriques sont sélectionnés (processus d'individualisation) par les individus et ainsi intégrés dans leur soi historique (conscience générationnelle). Selon nos résultats, l'événement collectif le plus important a été celui du 11 septembre 2001, soit la première guerre du XXI ${ }^{\mathrm{e}}$ siècle. Cet événement permet de mieux comprendre la société dans laquelle nous vivons, une société occidentale plus globalisée et déterritorialisée. Plus particulièrement,

Les événements du 11 septembre 2001 combinent deux traits majeurs de la globalisation contemporaine: d'un côté cet attentat est le fruit d'un mouvement terroriste transnational à idéologie religieuse, qui est en soi un effet pervers de la globalisation; de l'autre la présence enveloppante des NTI [nouvelles technologies de l'information] dans la vie quotidienne contemporaine des habitants de la planète (Lalive d'Epinay et coll., 2012, 13).

17 En se référant au tableau 2, le 11 septembre 2001 est l'événement le plus important pour les trois premières cohortes d'âges (20-24 ans : $29 \%$; 35-39 ans : $22 \%$; et 50-54 ans : $15 \%)$ et il est presque l'événement le plus cité pour la cohorte des 65-69 ans (7\%, alors que l'élection du Parti Québécois en 1976 arrive en premier avec $8 \%$ ). Ce tableau indique également une forte différenciation des événements sociohistoriques cités chez l'ensemble des classes d'âge. Par exemple, si nous additionnons les cinq premiers événements cités par ordre d'importance, nous ne franchissons pas la barre des $42 \%$ en moyenne (de $33 \%$ à $49 \%$ chez les différentes cohortes). De plus, à l'exception des cinq premiers événements, la très grande majorité des changements sociohistoriques recueille en moyenne moins de $2 \%$ des mentions. L'obligation de s'individualiser amène peut-être les individus à se "bricoler » un soi historique individualisé, mais autour des mêmes premières impressions de la vie (p. ex. : le 11 septembre 2001 ou la Deuxième Guerre mondiale). En d'autres mots, certains individus (plus particulièrement les jeunes cohortes) intègrent à leur soi historique un même événement collectif vécu collectivement, mais ensuite l'importance est accordée à des événements singuliers. Cette différenciation du soi historique des individus peut suggérer une individualisation influencée par la capacité de faire des choix libres à 
l'extérieur des normes sociales ou encore, des attributions de mémoires collectives. Cela dit, nos données empiriques ne permettent pas de confirmer en totalité cette observation. D'autres études, incluant des entrevues semi-dirigées, seront nécessaires afin de poursuivre la réflexion sur le processus d'individualisation et celui de la mémoire collective.

La persistance de mémoires nationales et l'émergence de la globalisation dans le soi historique. - Le tableau 3 présente les différents pays les plus cités en lien avec les événements sociohistoriques les plus frappants par catégorie d'âge. À l'exception des 20-24 ans, ce sont majoritairement des événements et des changements québécois qui arrivent au premier rang chez les catégories d'âges (de $34 \%$ à $48 \%$ ). Nonobstant le fait que le Québec est le plus cité chez quatre classes d'âge, l'ensemble des événements frappants s'est produit généralement à l'extérieur du Québec, et ce, d'une manière plus prononcée pour les cohortes âgées de moins de 39 ans (20-24 ans : $71 \%$; 35-39 ans: $66 \%$; $50-54$ ans : $57 \%$; 65-69 ans : $52 \%$; et 80-84 ans : $56 \%$ ). Notons que les États-Unis sont mentionnés majoritairement en tant que second pays (de $18 \%$ à $38 \%$ en moyenne), à l'exception des $80-84$ ans (seulement $5 \%$ des mentions). Pour cette cohorte, le Québec ( $44 \%)$ et le monde (36\%) sont les endroits les plus cités. Cela dit, l'émergence de la globalisation s'observe plus fortement dans le soi historique des cohortes nées durant la modernité avancée (depuis la fin des années 1960). Beck (2008) constate la présence des phénomènes de la globalisation et de la déterritorialisation dans les sociétés occidentales :

La détraditionalisation et la mise en place d'un réseau de médias mondial contribuent chaque jour davantage à détacher les parcours individuels de leurs cercles d'existence immédiats, et à les ouvrir à une télé-morale passant les frontières des pays et des domaines d'experts, qui place l'individu dans une situation durable de prise de position potentielle. [...] Tandis que les gouvernements agissent (encore) dans le cadre des États-nations, l'existence des individus s'ouvre déjà à la société mondiale (Beck, 2008, 294).

Nous vivons davantage dans une société mondiale. «Tous les pays, tous les groupes, sociaux, ethniques, familiaux, ont été amenés à connaître un profond changement du rapport traditionnel qu'ils entretenaient avec le passé » (Nora, 2002, 1). En somme, les différents pays mentionnés, à l'extérieur du territoire québécois, montrent l'importance de la globalisation et de la déterritorialisation dans le soi historique des individus. Les résultats présentés dans les tableaux 2 et 3 confirment l'hypothèse du soi historique davantage globalisé, à tout le moins, pour les plus jeunes catégories d'âge.

Paradoxalement, "en voulant désintégrer la mondialisation, Al-Qaïda stimule la formation d'une société-monde qui cherche à instituer sa police et sa gendarmerie et qui, mieux encore, pourrait - devrait - instituer une politique de civilisation pour la planète » (Morin, 2009, 21). Toujours d'une manière paradoxale, les réseaux terroristes mondiaux s'alimentent de la globalisation pour perpétuer leurs attaques antiglobalisation (Edmunds et Turner, 2005), mais elles contribuent aussi "à l'émergence de la société-monde » (Morin, 2009, 21). Edgar Morin (2009) n'est pas le seul intellectuel à imaginer le sort des humains dans une société-monde, car Ulrich Beck (2008) souhaite que les futurs citoyens soient plus cosmopolites dans des États et nations conçus à l'écart des définitions préétablies ou des legs historiques des identités nationales. Par exemple, la société-monde pourrait réguler les conflits ethniques et les guerres dans une politique de civilisation mondiale (Morin, 2009). Qui plus est, pour Amin Maalouf, la société-monde devrait permettre : 
Que personne ne se sente exclu de la civilisation commune qui est en train de naître, que chacun puisse y retrouver sa langue identitaire, et certains symboles de sa culture propre, que chacun, là encore, puisse s'identifier, ne serait-ce qu'un peu, à ce qu'il voit émerger dans le monde qui l'entoure, au lieu de chercher refuge dans un passé idéalisé (Maalouf, 1998, 210).

21 Nous pouvons faire un parallèle entre la réflexion de Maalouf et celle de Simmel. La thèse de Simmel (1999 [1908]) propose que « chaque individu, pour donner un sens à son existence et accepter la vie en société, doit être convaincu que sa personnalité singulière a une place spécifique dans le grand échiquier socio-économique de l'humanité » (Truc, 2005, 68). La société-monde doit chercher l'unité de l'humanité dans la diversité, c'est-à-dire "l'unité déployée » (Simmel, 1999 [1908], 702) dans la complémentarité des singularités (Truc, 2005). Bref, l'avènement de la société-monde doit s'enraciner dans la globalisation, mais également dans le cosmopolitisme et la valorisation du citoyen du monde (Mau, Mewes et Zimmermann, 2008). Ainsi, c'est l'étude dans la longue durée de la globalisation qui dira lesquels de ces intellectuels (Beck, Morin et Maalouf, entre autres) auront été des visionnaires de la modernité avancée et globalisée.

\section{Conclusion}

Le 11 septembre 2001 a influencé le soi historique de plusieurs individus que nous avons étudiés et a été une occasion d'approfondir le phénomène de la globalisation. De plus, cet événement a eu des impacts collatéraux importants (coûts économiques, humains, etc.) sur les Américains et en général, chez les pays occidentaux (la sécurité frontalière et internationale, la lutte contre le terrorisme, la guerre en Afghanistan, etc.). Ces attaques terroristes perpétrées sur le sol américain perturbent les autres pays également (phénomène de déterritorialisation et de globalisation), car le monde vit, avec l'Amérique, dans un réseau de relations partagées, comme l'ont d'ailleurs affirmé certains répondants.

Nous avons repéré que les cohortes les plus jeunes avaient été grandement frappées par les attaques terroristes aux États-Unis et que leur soi historique était davantage globalisé que celui des autres cohortes d'âge. Or, afin de bien comprendre les effets de la globalisation sur le soi historique des individus, certains auteurs tels que Beck et Beck-Gernsheim (2009) ainsi qu'Edmunds et Turner (2005), proposent d'étudier le concept de génération globalisée (Global Generation). En quelque sorte, faire l'expérience du 11 septembre 2001, "c'était faire partie de ce groupe, un groupe qui avait pris naissance en raison de l'événement lui-même » (Carr, 2010, 89). D’ailleurs, les nouvelles générations seraient davantage globalisées du fait qu'elles voyagent plus que les autres et qu'elles sont en constante interaction avec « la société-monde » ancrée, entre autres, dans les nouvelles technologies d'information et de communication (Edmunds et Turner, 2005). Cela dit, d'autres études sur l'émergence des générations globalisées seront nécessaires afin d'observer leur progression au sein de la modernité avancée.

Selon nous, ce n'est pas tant la fin de l'Histoire et celle des États-nations qui est en cause avec le 11 septembre 2001, mais il s'agit plutôt d'un événement catalyseur des forces antinomiques d'un même monde déterritorialisé et globalisé. Ainsi, les nouvelles dynamiques ethniques et culturelles, initiées par les forces de la globalisation, font émerger de nouvelles reconfigurations identitaires. Les médiascapes (télévision, 
Internet, téléphone, ordinateur, cinéma ; Appadurai, 2001) jouent un rôle indéniable dans la construction d'un soi historique globalisé. Chez les 20-24 ans, d'une manière assez précise avec les attentats du 11 septembre 2001, « on voit se dessiner une cohorte transnationale dont la mémoire historique se construit autour d'un événement qui symbolise à l'excès certains traits de la globalisation actuelle " (Lalive d'Epinay et coll., 2012, 7).

Nous avançons, en terminant, l'idée que l'individu est enraciné dans un contexte à la fois local et, peu ou prou, cosmopolite par l'essor des technologies de l'information et de communication, des réseaux sociaux et culturels. Les attaques terroristes du 11 septembre 2001 auront été un événement sociohistorique marquant pour plusieurs « individualistes » et « cosmopolites ».

\section{BIBLIOGRAPHY}

Appadurai, Arjun, Après le colonialisme. Les conséquences culturelles de la globalisation, Édition Payot \& Rivages, 2001.

Beck, Ulrich, La société du risque. Sur la voie d'une autre modernité, Paris, Éditions Flammarion, 2008.

Beck, Ulrich, «La société du risque globalisé revue sous l'angle de la menace terroriste », Cahiers internationaux de sociologie, 1, 114, 2003, 27-33.

Beck, Ulrich, The Brave New World of Work, Cambridge, Polity Press, 2000.

Beck, Ulrich et Beck-Gernsheim, Elisabeth, « Global Generations and the Trap of Methodological Nationalism for a Cosmopolitan Turn in the Sociology of Youth and Generation », European Sociological Review, 25, 1, 2009, 25-36.

Bensa, Alban et Fassin, Éric, 2002, « Les sciences sociales face à l'événement », Terrain, 38, 2002, 5-20, http://terrain.revues.org/1888 (page consultée le 20 septembre 2013).

Carr, David, «Y a-t-il une expérience directe de l'histoire ? La chute du mur de Berlin et le 11 septembre 2001 », A contrario, 1, 13, 2010, 83-94.

Deleuze, Gilles et Guattari, Félix, Mille Plateaux, Paris, Éditions de Minuit, 1980.

Dubet, François, Le travail des sociétés, Paris, Éditions du Seuil, 2009.

Dubet, François, « Pour une conception dialogique de l'individu », EspacesTemps.net., 2005, http://espacestemps.net/document1438.html (page consultée le 20 septembre 2013).

Edmunds, June et Turner, Bryan S., « Global Generations: Social Change in the Twentieth Century ", The British Journal of Sociology, 56, 4, 2005, 559-577.

Eisenstadt, Shmuel N., « Multiple Modernities », Daedalus, 129, 1, 2000, 1-29.

Fragnon, Julien, « Le 11-Septembre réécrit l'Histoire », Interrogations ?, I, 2005, 1-20, http:// www.revue-interrogations.org/fichiers/19/ 
le\%2011\%20septembre\%20reecrit\%20l\%20histoire\%20J\%20Fragnon.pdf (page consultée le 20 septembre 2013).

Giddens, Anthony, Les conséquences de la modernité, Paris, Éditions L’Harmattan, 1994.

Janssen, Steve M., Murre, Jaap M. J. et Meeter, Martijn, « Reminiscence Bump in Memory for Public Events », European Journal of Cognitive Psychology, 20, 4, 2008, 738-764.

Lalive d'Epinay, Christian, Concha, Victor, Gastron, Liliana, Guichard, Eduardo, Henriquez, Guillermo, Lynch, Gloria et Oddone, Maria Julieta, « Mondialisation et mémoires de l'histoire. Une comparaison internationale et intergénérationnelle. » Dans Rahma Bourqia, La sociologie et ses frontières. Faits et effets de la mondialisation, volume I, Paris, L'Harmattan, 2012.

Lalive d'Epinay, Christian et Cavalli, Stefano, « Mémoire de l'histoire et appartenance générationnelle des personnes âgées », Gérontologie et Société, 130, 2009, 127-144.

Lalive d'Epinay, Christian, Cavalli, Stefano, Aeby, Gaëlle, Gastron, Liliana, Oddone, Julieta, Lynch, Gloria et Lacasa, Debora, «Générations et mémoire historique. Une comparaison internationale. » Dans Didier Vrancken; et Laurence Thomsin, Le social à l'épreuve des parcours de vie, Louvain-la-Neuve, Brylant-Academia, 2008.

Maalouf, Amin, Les identités meurtrières, Paris, Les Éditions Bernard Grasset, 1998.

Mannheim, Karl, Le problème des générations, Paris, Nathan, 1990 [1928].

Martuccelli, Danilo, Forgé par l'épreuve. L'individu dans la France contemporaine, Paris, Armand Colin, 2006.

Mau, Steffen, Mewes, Jan et Zimmermann, Ann, « Cosmopolitan Attitudes through Transnational Social Practices? », Global Networks, 8, 1, 2008, 1-24.

Morin, Edgar, « Une mondialisation plurielle », Synergies, 6, 2009, 19-24.

Niemeyer, Katharina, « Le journal télévisé entre histoire, mémoire et historiographie », A contrario, 1, 13, 2010, 95-112.

Rousso, Henry, Vers une mondialisation de la mémoire, « Vingtième siècle. Revue d'histoire », 2, 94, 2007, 3-10.

Schmoll, Patrick, « Le 11 septembre : la fin de la modernité ? »Cultures en Mouvement, 44, 2002, 55-57.

Simmel, Georg, Sociologie, étude sur les formes de la socialisation, Paris, Presses Universitaires de France, [1908] 1999.

Sorensen, Mads P. et Christiansen, Allan, Ulrich Beck. An Introduction to the Theory of Second Modernity and the Risk Society, New York, Routledge, 2013.

Statistique Canada, « Recensement de 2006 », 2006 http://www12.statcan.ca/censusrecensement/2006/index-fra.cfm (page consultée le 20 septembre 2013).

Truc, Gérôme, « Simmel, sociologue du cosmopolitisme », Tumultes, 24, 2005, 49-77.

\section{NOTES}

1. «La perspective médiatique reproduit à l'infini l'image vertigineuse de la collision : c'est l'évidence aveuglante de l'instant qui fait l'événement » (Bensa et Fassin, 2002, 13).

2. Pour plus de détails, consultez le site de l'étude : http://www2.supsi.ch/cms/cevi/

3. Le questionnaire a été validé et répété dans plusieurs pays (cf. Lalive d'Epinay et coll., 2008). 
4. Cette forme de biais a été aussi rencontrée lors de l'étude suisse (Lalive d'Epinay et Cavalli, 2009) et dans d'autres études internationales portant sur le même thème de recherche (Janssen, Murre et Meeter, 2008).

5. René Lévesque est élu premier ministre du Québec (Parti Québécois) avec la promesse électorale de tenir un référendum sur l'indépendance du Québec.

\section{ABSTRACTS}

Everyone (or almost) remembers the fateful date associated with the collapsing Twin Towers of the World Trade Center in New York, the terrorist attacks in Washington and Pennsylvania State. In this article, we study in Quebec the impact and the signification of the September 11, 2011 among different age cohorts. In this way, we would see the importance of the globalization in their historical self. Based on a mixed-methods research design, the study involved 504 adults from Quebec City participated in the CEVI international study (Changes and Events across the Life Course). The quantitative results of socio-historical changes show the importance of the September 11 attacks, reflecting the trends of the globalization of collective memories. This event affected the historical self of many people that we studied and was an occasion to deepen the phenomenon of globalization.

Tout le monde (ou presque) se souvient de cette date fatidique associée à la chute des deux tours du World Trade Center de New York, des attaques terroristes à Washington et en Pennsylvanie. Dans cet article, nous étudions au Québec l'impact et la signification du 11 septembre $2001 \mathrm{chez}$ différentes cohortes d'âge. De cette manière, nous observons l'importance de la globalisation dans leur soi historique. Selon une méthodologie mixte de la recherche, 504 adultes de la Ville de Québec ont participé à l'étude internationale CEVI (Changements et événements au cours de la vie). L'analyse quantitative des changements sociohistoriques montre l'importance des attaques terroristes du 11 septembre 2001, signe d'une tendance à la globalisation des mémoires collectives. Cet événement a influencé le soi historique de plusieurs individus que nous avons étudiés et a été une occasion d'approfondir le phénomène de la globalisation.

\section{INDEX}

Mots-clés: 11 septembre 2001 ; soi historique ; mémoire collective ; Québec ; Globalisation

Keywords: September 11, 2001; historical self; collective memory; Quebec; Globalization

\section{AUTHOR}

\section{CHRISTIAN BERGERON}

Professeur auxiliaire, École interdisciplinaire des sciences de la santé, Université d'Ottawa, Ontario, Canada 